\title{
Maximum Likelihood Estimation of the Lognormal-Rician FSO Channel Model
}

\author{
Luanxia Yang, Julian Cheng, Senior Member, IEEE, and Jonathan F. Holzman, Member, IEEE
}

\begin{abstract}
In this work, the on-going challenges are addressed for the application of the lognormal-Rician turbulence model to free-space optical communication systems. Maximum likelihood estimation is applied to characterize the lognormal-Rician turbulence model parameters, and the expectation-maximization algorithm is used to compute maximum likelihood estimates of the unknown parameters. The performance is investigated, by way of the mean square error, and it is found that the proposed technique can accurately characterize free-space optical communication channels over a wide range of turbulence conditions, with reduced demand on the quantity of data samples.
\end{abstract}

Index Terms-Free-space optical communications, lognormalRician turbulence model, maximum likelihood estimation.

\section{INTRODUCTION}

Free-space optical (FSO) communication, also known as outdoor optical wireless communication, is a broadband wireless technology that provides high-speed data connectivity between nodes of optical line-of-sight links. High-speed data transmission is facilitated by modulated optical beams through the atmosphere. Such FSO systems offer several benefits, including cost-effectiveness, rapid deployment, enhanced security, protocol transparency, and freedom from spectral licensing regulations [1]. However, optical signals transmitted over free-space are subject to distortion due to transient inhomogeneities of atmospheric temperature and pressure [2]. The resulting scintillation or fading is a major cause of performance degradation for FSO systems.

Current FSO systems often use intensity modulation and direct detection (IM/DD) with on-off keying (OOK) and fixed detection thresholds for reasons of practicality [3]. However, such fixed and unoptimized detection thresholds can result in irreducible error floors in large signal-to-noise ratio (SNR) regimes [4]. Attempts to overcome the irreducible error floors of OOK IM/DD systems have focused on the application of adaptive detection thresholds.

Adaptive detection of OOK signal can be classified into three categories. The first category, idealized adaptive detection, applies bit-by-bit adaptations to the detection threshold on the (typically) nanosecond timescale of the bit interval [2], [5]. The second category, quasi-static adaptive detection, applies adaptations to the detection threshold on the (typically) millisecond timescale of the turbulence coherence time [6]. These adaptations can be difficult to implement-particularly for FSO receivers using numerous photodetectors. The third category, electrical-SNR-optimized detection [7], [8], unlike

The authors are with the School of Engineering, The University of British Columbia, Kelowna, BC, Canada (e-mail: \{luanxia.yang, julian.cheng, jonathan.holzman\}@ubc.ca). the prior two categories, does not require knowledge of the instantaneous channel state information. Once the detection threshold is optimized with respect to the electrical SNR, the receiver operates with a fixed detection threshold, and this continues while the turbulence channel exhibits stationary statistics, i.e., constant channel model parameters, which is typically on the timescale of several minutes [9]. Thus, electrical-SNR-optimized detection offers the practical advantages of operation with fixed detection thresholds (by avoiding the need for rapid adaptations) and the performance advantages of operation with idealized adaptive detection thresholds (by eliminating irreducible error floors). It must be noted, however, that the success of such systems relies heavily upon accurate statistical models of the FSO fading channels. The work that follows addresses this challenge of channel parameter estimation for OOK IM/DD systems.

Many statistical models have been proposed to describe FSO fading channels. There exist models for use with a specific range of turbulence conditions: the lognormal distribution characterizes FSO fading channels under weak turbulence conditions over meters (or longer, depending on the temperature, wind strength, altitude, humidity, and atmospheric pressure) [9], [10]; the K-distribution characterizes FSO fading channels under strong turbulence conditions over several kilometers [11]; the negative exponential distribution characterizes FSO fading channels in the limiting case of saturated scintillation [12]. There also exist generalized models for use over a broad range of weak-to-strong turbulence conditions: the GammaGamma distribution, which can underestimate effects of smalland large-scale scintillations and suffer from decreased accuracy [13], and the lognormal-Rician distribution, which has been found to offer two advantages. First, heuristic analyses of wave propagation through turbulence show that the lognormal-Rician fading distribution accurately characterizes experimental data [14]. Second, the lognormal-Rician fading distribution is highly adaptable over a wide range of weak-tostrong turbulence conditions through its parameters. However, the application of the lognormal-Rician fading distribution has been limited, as it does not have a tractable closed-form probability density function (pdf).

Given the potential of the lognormal-Rician distribution, for accurately characterizing FSO fading channels, there have been efforts to implement this distribution with estimated shaping parameters. In [15], the authors applied a physical model of turbulence-induced scattering, to estimate the shaping parameters of the lognormal-Rician fading distribution. It should be noted, however, that this approach depends heavily upon estimated parameters in a physical model of the 
turbulence-induced scattering and such parameters are often either unavailable or lacking in accuracy. For computational simplicity, the authors applied the Tatarskii model to characterize refractive index fluctuations and geometrical optics to characterize turbulent eddies, but the underlying assumptions of this approach can lead to notable inaccuracies, as discussed in [14]. In [16], the authors introduced the generalized method of moments approach to estimate the shaping parameters of the lognormal-Rician distribution. It should be noted, however, that this approach demands a large number of data samples, on the order of $10^{6}$ data samples, and this impedes its implementation in FSO communications. For a standard FSO link, experiencing quasi-static turbulence fading on a typical millisecond timescale, the system would exhibit latency on the order of $1 \times 10^{6}$ milliseconds $=1000$ seconds. This duration is unacceptably long for FSO communications, as typical FSO channels exhibit stationary statistics, i.e., constant channel model parameters, on the timescale of several minutes. Clearly, FSO systems applying channel estimation with a lognormalRician fading distribution need a more rapid estimation of the shaping parameters, to witness its benefits.

In this paper, we propose the use of maximum likelihood estimation (MLE), with the expectation-maximization (EM) algorithm, to estimate the parameters of the lognormal-Rician distribution. The estimation approach is found to be highly accurate, and it operates with relatively low quantities of data samples (on the order of one thousand data samples).

\section{LOGNORMAL-RICIAN PROBABILITY DENSITY FUNCTION}

For the FSO system of interest, there is an assumption of perfect background noise rejection, from narrowband optical, electronic, and/or spatial filtering [17]- [19]. For the resulting lognormal-Rician channel model, the optical irradiance $I$ can be obtained by $I=\left|U_{C}+U_{G}\right|^{2} \exp (2 \chi)$, where $U_{C}$ is a real deterministic quantity, $U_{G}$ is a circular complex Gaussian random variable (RV) with zero mean, $\chi$ is a real Gaussian $\mathrm{RV},\left|U_{C}+U_{G}\right|$ is a Rician RV, $\left|U_{C}+U_{G}\right|^{2}$ is a noncentral chisquare RV with a degree of freedom of two, and $\exp (2 \chi)$ is a lognormal RV. Consequently, $I$ follows a lognormal-Rician distribution with a pdf given by [15]

$$
\begin{aligned}
f_{I}(I)= & \frac{(1+r) e^{-r}}{\sqrt{2 \pi} \sigma_{z}} \int_{0}^{\infty} \frac{d z}{z^{2}} I_{0}\left(2\left[\frac{(1+r) r}{z} I\right]^{1 / 2}\right) \\
& \times \exp \left(-\frac{1+r}{z} I-\frac{1}{2 \sigma_{z}^{2}}\left(\ln z+\frac{1}{2} \sigma_{z}^{2}\right)^{2}\right)
\end{aligned}
$$

where $z$ represents $\exp (2 \chi), r=\left|U_{C}\right|^{2} / E\left[\left|U_{G}\right|^{2}\right]$ is the coherence parameter, $E[\cdot]$ denotes the expectation operation, $\sigma_{z}^{2}$ is the variance of the logarithm of the irradiance modulation factor $z$, and $I_{0}(\cdot)$ is the zero-order modified Bessel function of the first kind. As noted on [13], it is not generally known how to directly relate these two empirical parameters to the physical characteristics of atmospheric conditions, but it is possible to characterize trends in the two parameters, with respect to the Rytov variance, $\sigma_{R}^{2}=0.5 k^{\frac{7}{6}} L^{\frac{11}{6}} C_{n}^{2}$. Here, $k=2 \pi / \lambda$ is the wavenumber for the wavelength of $\lambda, L$ is the link length, and
$C_{n}^{2}$ is the altitude-dependent index of the refractive structure parameter, varying from $10^{-17} \mathrm{~m}^{-\frac{2}{3}}$ for weak turbulence to $10^{-13} \mathrm{~m}^{-\frac{2}{3}}$ for strong turbulence. The characteristic trends in the two parameters are seen in [15], for variations in the Rytov variance, $\sigma_{R}^{2}$, i.e., variations in $L$ and/or $C_{n}^{2}$. In the limit of zero inner scale, it is shown that the parameter $r$ decreases as $\sigma_{R}^{2}$ increases, while the parameter $\sigma_{z}^{2}$ is approximately equal to the Rytov variance for small $\sigma_{R}^{2}$, reaches a peak value of approximately 0.58 for $\sigma_{R}^{2} \approx 8$, and decreases slowly to approximately 0.4 for large $\sigma_{R}^{2}$.

\section{MLE FOR THE LOGNORMAL-RICIAN SHAPING PARAMETER ESTIMATION}

The maximum likelihood principle is the most popular approach to obtaining practical estimators. Its performance is optimal for large quantities of data, and it yields an approximation of the minimum-variance unbiased estimator. The EM algorithm is applied to compute the MLE of the lognormal-Rician parameters.

Assuming we have $K$ independent and identically distributed observations of the lognormal-Rician distribution, $\boldsymbol{I}=\left[\begin{array}{lll}I[0] & \ldots & I[K-1\end{array}\right]^{T}$, with the unknown vectors $\boldsymbol{\theta}=$ $\left[\begin{array}{ll}\sigma_{z}^{2} & r\end{array}\right]^{T}$, the pdf of $\boldsymbol{I}$ can be written as

$$
\begin{aligned}
f_{\boldsymbol{I}}(\boldsymbol{I} ; \boldsymbol{\theta}) & =\prod_{l=0}^{K-1} \frac{(1+r) e^{-r}}{\sqrt{2 \pi} \sigma_{z}} \int_{0}^{\infty} I_{0}\left(2\left[\frac{(1+r) r}{z} I[l]\right]^{1 / 2}\right) \\
& \times \exp \left(-\frac{1+r}{z} I[l]-\frac{1}{2 \sigma_{z}^{2}}\left(\ln z+\frac{1}{2} \sigma_{z}^{2}\right)^{2}\right) \frac{d z}{z^{2}}
\end{aligned}
$$

The MLE of the unknown vector $\boldsymbol{\theta}$ is then obtained by maximizing the $\log$-likelihood function

$$
\begin{aligned}
& L(\boldsymbol{I} ; \boldsymbol{\theta})=\ln f(\boldsymbol{I} ; \boldsymbol{\theta}) \\
& =\sum_{l=0}^{K-1} \ln \left(\frac{(1+r) e^{-r}}{\sqrt{2 \pi} \sigma_{z}} \int_{0}^{\infty} I_{0}\left(2\left[\frac{(1+r) r}{z} I[l]\right]^{1 / 2}\right)\right. \\
& \left.\quad \times \exp \left(-\frac{1+r}{z} I[l]-\frac{1}{2 \sigma_{z}^{2}}\left(\ln z+\frac{1}{2} \sigma_{z}^{2}\right)^{2}\right)\right) \frac{d z}{z^{2}} .
\end{aligned}
$$

It is difficult to obtain closed-form estimates of the lognormal-Rician parameters, due to the integral form of (12), so the following analysis uses the EM algorithm to find the MLE of $\boldsymbol{\theta}$ [21]. Given $\boldsymbol{z}=\left[\begin{array}{lll}z[0] & \ldots & z[L-1]]^{T} \text { as }\end{array}\right.$ the unobserved data, we can write the complete-data loglikelihood function as

$$
\begin{aligned}
& L(\boldsymbol{I}, \boldsymbol{z} ; \boldsymbol{\theta})=\sum_{l=0}^{K-1} \ln f\left(z[l] ; \sigma_{z}\right)+\sum_{l=0}^{K-1} \ln f(I[l] \mid z[l] ; r) \\
& =K\left\{-\ln \sqrt{\left(2 \pi \sigma_{z}^{2}\right)}+\ln (1+r)-\frac{5}{2 K} \sum_{l=0}^{K-1} \ln z[l]\right. \\
& -\frac{\sigma_{z}^{2}}{8}-\frac{1}{2 \sigma_{z}^{2}} \frac{1}{K} \sum_{l=0}^{K-1}(\ln z[l])^{2}-\frac{1}{K} \sum_{l=0}^{K-1} \frac{1+r}{z[l]} I[l] \\
& \left.-r+\frac{1}{K} \sum_{l=0}^{K-1} \ln I_{0}\left(2\left[\frac{(1+r) r}{z[l]} I[l]\right]^{1 / 2}\right)\right\} .
\end{aligned}
$$


where $f\left(z[l] ; \sigma_{z}\right)=\frac{1}{\sqrt{2 \pi} \sigma_{z} z[l]} \exp \left(-\frac{1}{2 \sigma_{z}^{2}}\left(\ln z[l]+\frac{1}{2} \sigma_{z}^{2}\right)^{2}\right)$ and $f(I[l] \mid z[l] ; r)=\frac{1+r}{z[l]} I_{0}\left(2\left[\frac{(1+r) r}{z[l]} I[l]\right]^{1 / 2}\right) \exp (-r$ $\left.-\frac{1+r}{z[l]} I[l]\right)$. The resulting complete-data sufficient statistics are $T_{1}(\boldsymbol{z})=\frac{1}{K} \sum_{l=0}^{K-1} \ln z[l], \quad T_{2}(\boldsymbol{z})=$ $\frac{1}{K} \sum_{l=0}^{K-1}(\ln z[l])^{2}$, and $T_{3}(\boldsymbol{z})=-\frac{1}{K} \sum_{l=0}^{K-1} \frac{1+r}{z[l]} I[l]+$ $\frac{1}{K} \sum_{l=0}^{K-1} \ln I_{0}\left(2\left[\frac{(1+r) r}{z[l]} I[l]\right]^{1 / 2}\right)$.

The initial value of $\sigma_{z}^{2}$ is obtained by fitting the simple lognormal turbulence model, where ${\hat{\sigma_{z}^{2}}}^{(0)}=-\frac{2}{K} \sum_{l=0}^{K-1} \ln I[l]$. The initial value of the coherence parameter $r$ can be obtained by solving the polynomial equation [16, eq. (6)]

$$
\begin{aligned}
& \frac{\left[\left(\hat{r}^{(0)}\right)^{2}+4 \hat{r}^{(0)}+2\right]^{3}}{\left[1+\left(\hat{r}^{(0)}\right)^{3}\right]\left[\left(\hat{r}^{(0)}\right)^{3}+9\left(\hat{r}^{(0)}\right)^{2}+18 \hat{r}^{(0)}+6\right]} \\
& =\frac{\left(\frac{1}{K} \sum_{l=0}^{K-1} I^{2}[l]\right)^{3}}{\frac{1}{K} \sum_{l=0}^{K-1} I^{3}[l]} .
\end{aligned}
$$

Iterations are then carried out with the expectation-step (Estep) and maximization-step (M-step).

E-step: The E-step is carried out by computing

$$
\begin{aligned}
& T_{1}\left(\boldsymbol{z} ; \hat{\boldsymbol{\theta}}^{(j)}\right)=\frac{1}{K} \sum_{l=0}^{K-1} E_{\boldsymbol{z} \mid \boldsymbol{I}}\left[\ln z[l] \mid \boldsymbol{I} ; \hat{\boldsymbol{\theta}}^{(j)}\right] \\
& T_{2}\left(\boldsymbol{z} ; \hat{\boldsymbol{\theta}}^{(j)}\right)=\frac{1}{K} \sum_{l=0}^{K-1} E_{\boldsymbol{z} \mid \boldsymbol{I}}\left[(\ln z[l])^{2} \mid \boldsymbol{I} ; \hat{\boldsymbol{\theta}}^{(j)}\right] \\
& T_{3}\left(\boldsymbol{z} ; \hat{\boldsymbol{\theta}}^{(j)}\right)=-\frac{1}{K} \sum_{l=0}^{K-1}(1+r) E_{\boldsymbol{z} \mid \boldsymbol{I}}\left[z^{-1}[l] \mid \boldsymbol{I} ; \hat{\boldsymbol{\theta}}^{(j)}\right] I[l] \\
& +\frac{1}{K} \sum_{l=0}^{K-1} E_{\boldsymbol{z} \mid \boldsymbol{I}}\left[\ln I_{0}\left(2\left[\frac{(1+r) r}{z[l]} I[l]\right]^{1 / 2}\right) \mid \boldsymbol{I} ; \hat{\boldsymbol{\theta}}^{(j)}\right]
\end{aligned}
$$

where $\hat{\boldsymbol{\theta}}^{(j)}=\left[{\hat{\sigma_{z}^{2}}}^{(j)} \hat{r}^{(j)}\right]^{T}$ is an estimate of $\boldsymbol{\theta}$ in the $j$ th iteration. For the computations carried out as part of the Estep, it is noted that the expectation expressions in (8)-(10) are all functions of $z[l]$, so the conditional expectations in (8)-(10) can be expressed as

$$
E_{\boldsymbol{z} \mid \boldsymbol{I}}\left[g(z[l]) \mid \boldsymbol{I} ; \hat{\boldsymbol{\theta}}^{(j)}\right]=\int g(z[l]) f\left(z[l] \mid \boldsymbol{I} ; \hat{\boldsymbol{\theta}}^{(j)}\right) d z[l]
$$

where $f\left(z[l] \mid I[l] ; \hat{\boldsymbol{\theta}}^{(j)}\right)=\frac{f\left(I[l] \mid z[l] ; \hat{\boldsymbol{\theta}}^{(j)}\right) f\left(z[l] ; \hat{\boldsymbol{\theta}}^{(j)}\right)}{f\left(I[l] ; \hat{\boldsymbol{\theta}}^{(j)}\right)}$ and where

$$
\begin{aligned}
& f\left(I[l] \mid z[l] ; \hat{\boldsymbol{\theta}}^{(j)}\right)=\exp \left(-\hat{r}^{(j)}-\frac{1+\hat{r}^{(j)}}{z[l]} I[l]\right) \\
& \times \frac{1+\hat{r}^{(j)}}{z[l]} I_{0}\left(2\left[\frac{\left(1+\hat{r}^{(j)}\right) \hat{r}^{(j)}}{z[l]} I[l]\right]^{1 / 2}\right) \\
& f\left(z[l] ; \hat{\boldsymbol{\theta}}^{(j)}\right)=\frac{1}{\sqrt{2 \pi} \hat{\sigma}_{z}^{(j)} z[l]} \\
& \times \exp \left(-\frac{1}{2\left(\hat{\sigma}_{z}^{(j)}\right)^{2}}\left[\ln z[l]+\frac{1}{2}\left(\hat{\sigma}_{z}^{(j)}\right)^{2}\right]^{2}\right)
\end{aligned}
$$

and

$$
\begin{aligned}
& f\left(I[l] ; \hat{\boldsymbol{\theta}}^{(j)}\right)=\frac{\left(1+\hat{r}^{(j)}\right) e^{-\hat{r}^{(j)}}}{\sqrt{2 \pi} \hat{\sigma}_{z}^{(j)}} \int_{0}^{\infty} I_{0}\left(2\left[\frac{\left(1+\hat{r}^{(j)}\right) \hat{r}^{(j)}}{z[l]} I[l]\right]^{1 / 2}\right) \\
& \times \exp \left(-\frac{1+r}{z[l]} I[l]-\frac{1}{2\left(\hat{\sigma}_{z}^{(j)}\right)^{2}}\left[\ln z[l]+\frac{1}{2}\left(\hat{\sigma}_{z}^{(j)}\right)^{2}\right]^{2}\right) \frac{d z}{z[l]^{2}} .
\end{aligned}
$$

M-step: The M-step is carried out by computing

$$
{\hat{\sigma_{z}^{2}}}^{(j+1)}=T_{2}\left(\boldsymbol{z} ; \hat{\boldsymbol{\theta}}^{(j)}\right)-\left(T_{1}\left(\boldsymbol{z} ; \hat{\boldsymbol{\theta}}^{(j)}\right)\right)^{2}
$$

and finding $\hat{r}^{(j+1)}$ such that it maximizes $\hat{r}^{(j+1)}=\arg \max _{r}\left\{T_{3}\left(\boldsymbol{z} ; \hat{\boldsymbol{\theta}}^{(j)}\right)\right\}$, where $\hat{\boldsymbol{\theta}}^{(j+1)}=$ $\left[{\hat{\sigma_{z}^{2}}}^{(j+1)} \hat{r}^{(j+1)}\right]^{T}$ is the new estimate of $\boldsymbol{\theta}$. For the EM algorithm, the conditional expectation of the complete data is nondecreasing until it reaches a fixed point. This fixed point is the MLE of $\boldsymbol{\theta}$, i.e., $\hat{\boldsymbol{\theta}}_{M L}=\left[\begin{array}{ll}\hat{\sigma}_{z, M L}^{2} & \hat{r}_{M L}\end{array}\right]^{T}$.

\section{NUMERICAL RESUlTS}

To evaluate the estimator performance, the mean squared error (MSE) of the estimator $\hat{\boldsymbol{\theta}}$ is studied, the MSE is $\operatorname{MSE}[\hat{\boldsymbol{\theta}}]=\operatorname{Var}[\hat{\boldsymbol{\theta}}]+(E[\hat{\boldsymbol{\theta}}]-\boldsymbol{\theta})^{2}$, where $\operatorname{Var}[\hat{\boldsymbol{\theta}}]$ is the variance of the estimator, i.e., $\operatorname{Var}[\hat{\boldsymbol{\theta}}]=\frac{1}{M-1} \sum_{i=0}^{M-1}\left(\hat{\boldsymbol{\theta}}_{i}-\overline{\hat{\boldsymbol{\theta}}}\right)^{2}, \overline{\hat{\boldsymbol{\theta}}}$ is the sample mean of the estimator, and $E[\hat{\boldsymbol{\theta}}]=\frac{1}{M} \sum_{i=0}^{M-1} \hat{\boldsymbol{\theta}}_{i}$ is the mean of the estimator [22]. The simulation uses $K=1,000$ data samples to estimate the lognormal-Rician parameters and $M=100$ trials to calculate the MSE of the estimator.

In Fig. 1, we present the simulated MSE and normalized mean squared error (NMSE) performance of $\hat{r}$ and $\hat{\sigma}_{z}^{2}$ when $\sigma_{z}^{2}=0.25$ and $r$ ranges from 1 to 9 . The NMSE is defined as the MSE scaled by the true value of the estimator. The performance trends at or above $r=2$ are especially noteworthy. Increasing the value of $r$ decreases the NMSE of $\hat{r}$ but it does not change the NMSE of $\hat{\sigma}_{z}^{2}$ to any great extent. Thus, changes to the value of $r$ have minimal effects on the estimation performance of $\sigma_{z}^{2}$, and the MLE is insensitive to the value of $r$. The same conclusion can be seen for the MSE of $\hat{r}$ and $\hat{\sigma}_{z}^{2}$, which remain relatively flat as $r$ increases.

In Fig. 2, we present the simulated MSE and NMSE performance of $\hat{r}$ and $\hat{\sigma}_{z}^{2}$ when $r=4$ and $\sigma_{z}^{2}$ ranges from 0.1 to 0.8 . From the figure, we note that the MSE of $\hat{\sigma}_{z}^{2}$ increases with the value of $\sigma_{z}^{2}$ while the MSE performance curve of $\hat{r}$ stays flat with changing values of $\sigma_{z}^{2}$. It can be seen that the MLE performance of the lognormal-Rician parameter $\sigma_{z}^{2}$ is insensitive to the value of $r$ but sensitive to the value of $\sigma_{z}^{2}$, while the MLE performance of the lognormal-Rician parameter $r$ is insensitive to both the values of $r$ and $\sigma_{z}^{2}$.

Overall, the results for the MSE and NMSE in Figs. 1 and 2 are indicative of accurate estimation. The MSE and NMSE performance is comparable with that of the prior study [16], albeit with three orders of magnitude fewer data samples. However, for the implementation of the proposed estimation technique, it is worth noting the underlying assumption above for perfect background noise rejection. In general, the MSE performance worsens with increasing background noise power, 


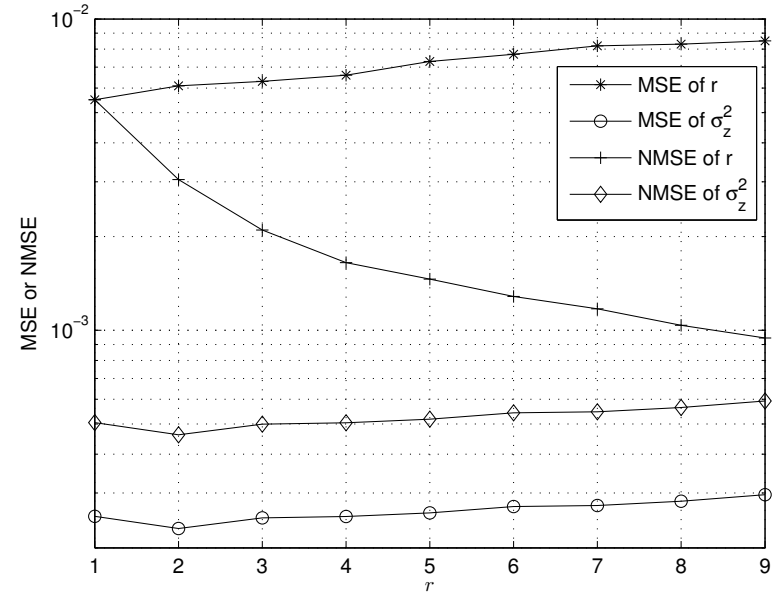

Fig. 1. MSE and NMSE performance of the maximum likelihood estimators for the lognormal-Rician parameters $r$ and $\sigma_{z}^{2}$ with $\sigma_{z}^{2}=0.25$.

so it is necessary to implement the technique in a system with a sufficient level of background noise rejection (to establish the desired level of MSE performance). For example, using the Monte-Carlo simulation, it can be shown that the MSE for the estimated parameters can be maintained below 0.1 or below 0.01 , by rejecting the background noise power to levels below $4 \%$ or below $0.5 \%$ of the signal power, respectively. Such background noise rejection can be achieved through established techniques of optical, electronic, and spatial filtering [17]- [19].

\section{CONCLUSION}

In this work, the challenges were addressed for the application of the lognormal-Rician turbulence model to FSO communications. The proposed technique used MLE, to estimate the parameters of the lognormal-Rician fading channels and the EM algorithm, to compute the MLE of the unknown parameters. The performance was simulated in terms of MSE. Numerical results showed that MLE with the EM algorithm can effectively characterize FSO communications over lognormal-Rician fading channels, given a wide range of turbulence conditions. Accurate estimation was shown with reduced demands on the quantity of data samples.

\section{REFERENCES}

[1] V. W. S. Chan, "Free-space optical communications," IEEE/OSA J. Lightw. Technol., vol. 24, pp. 4750-4762, Dec. 2006.

[2] J. H. Shapiro and R. C. Harney, "Burst-mode atmospheric optical communication," in Proc.1980 Nat. Telecommun. Conf., Houston, TX, 1980, pp. 27.5.1-27.5.7.

[3] N. Chand, J. J. Loriz, A. J. Hunton, and B. M. Eteson, "Performance comparison of NRZ and RZ modulations with and without forward error corrections for free-space optical communication," Proc. SPIE, vol. 5892, pp. 58920U-1-58920U-8, Sept. 2005.

[4] J. Li, J. Q. Liu, and D. P. Taylor, "Optical communication using subcarrier PSK intensity modulation through atmospheric turbulence channels," IEEE Trans. Commun., vol. 55, pp. 1598-1606, Aug. 2007.

[5] M. Tycz, M. W. Fitzmaurice, and D. A. Premo, "Optical communication system performance with tracking error induced signal fading," IEEE Trans. Commun., vol. 21, pp. 1069-1072, Sept. 1973.

[6] M. L. B. Riediger, R. Schober, and L. Lampe, "Blind detection of onoff keying for free-space optical communications," in Proc. CCECE, Niagara Falls, Canada, 2008, pp. 1361-1364.

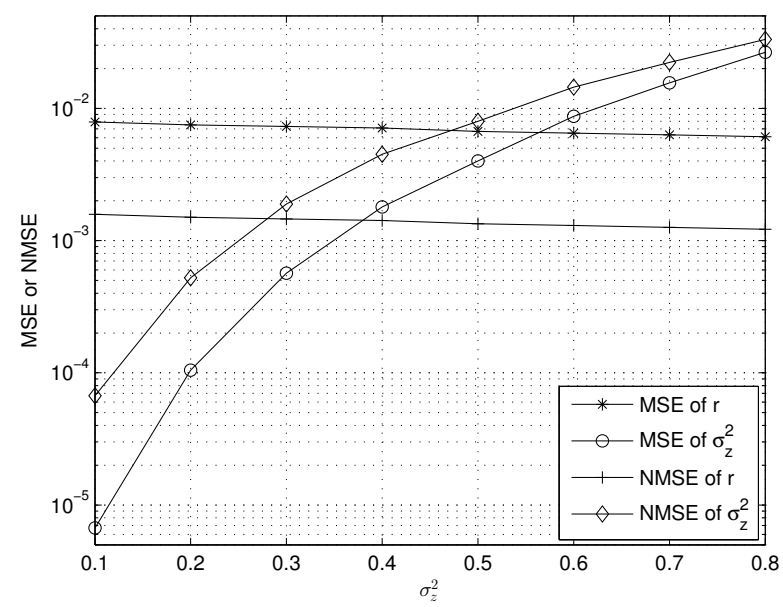

Fig. 2. MSE and NMSE performance of the maximum likelihood estimators for the lognormal-Rician parameters $r$ and $\sigma_{z}^{2}$ with $r=4$.

[7] X. Zhu and J. M. Kahn, "Free-space optical communication through atmospheric turbulence channels," IEEE Trans. Commun., vol. 50, pp. 1293-1300, Oct. 2002.

[8] L.Yang, J. Cheng, and J. F. Holzman, "Electrical-SNR-optimized detection threshold for OOK IM/DD optical wireless communications," in Proc. Canadian Workshop Inform. Theory, Toronto, ON, 2013, pp. 186-189.

[9] D. A. DeWolf, "Are strong irradiance fluctuations log normal or Rayleigh distributed," J. Opt. Soc. Amer., vol. 57, pp. 787-797, Jun. 1967.

[10] A. Prokeš, "Modeling of atmospheric turbulence effect on terrestrial FSO link," Radioengr., vol. 18, pp. 42-47, Apr. 2009.

[11] M. Niu, J. Cheng, and J. F. Holzman, "Exact error rate analysis of equal gain and selection diversity for coherent free-space optical systems on strong turbulence channels," Opt. Express, vol. 18, pp. 13915-13926, Jun. 2010.

[12] K. P. Peppas, F. Lazarakis, A. Alexandridis, and K. Dangakis, "Simple, accurate formula for the average bit error probability of multipleinput multiple-output free-space optical links over negative exponential turbulence channels," Opt. Lett., vol. 37, pp. 3243-3245, Aug. 2012.

[13] L. C. Andrews, R. L. Phillips, and C. Y. Hopen, Laser Beam Scintillation with Applications. Bellingham, WA: SPIE Press, 2001.

[14] R. J. Hill and R. G. Frehlich, "Probability distribution of irradiance for the onset of strong scintillation," J. Opt. Soc. Am. A, vol. 14, pp. 1530$1540,1997$.

[15] J. H. Churnside and S. F. Clifford, "Log-normal Rician probabilitydensity function of optical scintillations in the turbulent atmosphere," J. Opt. Soc. Am. A, vol. 4, 1923-1930, Oct. 1987.

[16] X. Song and J. Cheng, "Joint estimation of the lognormal-Rician atmospheric turbulence model by the generalized method of moments," Opt. Commun., vol. 285, pp. 4727-4732, Nov. 2012.

[17] J. Lumeau, V. Smirnov, A. Glebov, and L. B. Glebov, "Ultra-narrow bandpass filters based on volume Bragg grating technologies," Proc. SPIE, vol. 7675, pp. 76750H-1-76750H-9, Apr. 2010.

[18] J. M Senior, Optical Fiber Communications Principles and Practice, 3rd ed. Pearson Education Limited: Prentice Hall, 2009.

[19] S. Lee, K. E. Wilson, and M. Troy, "Background noise mitigation in deep-space optical communications using adaptive optics", IPN Prog. Rep., vol. 42-161, pp. 1-16, May 2005.

[20] J. H. Churnside and R. J. Hill, "Probability density of irradiance scintillations for strong path-integrated refractive turbulence," J. Opt. Soc. Am. A, vol. 4, pp. 727-733, Apr. 1987.

[21] A. Dogandǔić and J. Jin, "Maximum likelihood estimation of statistical properties of composite gamma-lognormal fading channel," IEEE Trans. Signal Proc., vol. 52, pp. 2940-2945, Oct. 2004.

[22] D. R. Pauluzzi and N. C. Beaulieu, "A comparison of SNR estimation techniques for the AWGN channel," IEEE Trans. Commun., vol. 48, pp. 1681-1691, Oct. 2000. 